\title{
Antimicrobial Stewardship in the Intensive Care Unit: The Role of Biomarkers, Pharmacokinetics, and Pharmacodynamics
}

\author{
Patrícia Moniz · Luís Coelho · Pedro Póvoa
}

Received: September 22, 2020 / Accepted: October 31, 2020 / Published online: November 20, 2020

(C) The Author(s) 2020

\section{ABSTRACT}

The high prevalence of infectious diseases in the intensive care unit (ICU) and consequently elevated pressure for immediate and effective treatment have led to increased antimicrobial therapy consumption and misuse. Moreover, the emerging global threat of antimicrobial resistance and lack of novel antimicrobials justify the implementation of judicious antimicrobial stewardship programs (ASP) in the ICU. However, even though the importance of ASP is generally accepted, its implementation in the ICU is far from optimal and current evidence regarding strategies such as de-escalation remains controversial. The limitations of

P. Moniz · L. Coelho · P. Póvoa ( $\square)$

Polyvalent Intensive Care Unit, Sao Francisco Xavier

Hospital, CHLO, Lisbon, Portugal

e-mail: pedrorpovoa@gmail.com

L. Coelho $\cdot$ P. Póvoa

Nova Medical School, CHRC, New University of

Lisbon, Lisbon, Portugal

P. Póvoa

Center for Clinical Epidemiology and Research Unit of Clinical Epidemiology, OUH Odense University

Hospital, Odense, Denmark clinical guidance for antimicrobial therapy initiation and discontinuation have led to multiple studies for the evaluation of more objective tools, such as biomarkers as adjuncts for ASP. C-reactive protein and procalcitonin can be adequate for clinical use in acute infectious diseases, the latter being the most studied for ASP purposes. Although promising, current evidence highlights challenges in biomarker application and interpretation. Furthermore, the physiological alterations in the critically ill render pharmacokinetics and pharmacodynamics crucial parameters for adequate antimicrobial therapy use. Individual pharmacokinetic and pharmacodynamic targets can reduce antimicrobial therapy misuse and risk of antimicrobial resistance.

Keywords: Antimicrobial stewardship; Biomarkers; De-escalation; Pharmacodynamics; Pharmacokinetics 


\section{Key Summary Points}

Antimicrobial stewardship program implementation remains suboptimal in the intensive care setting, where frequent prescription of broad-spectrum antimicrobial therapy and its misuse pose significant risk of antimicrobial resistance, adverse effects, and economic burden.

Biomarkers can be considered promising adjuncts for antimicrobial stewardship but knowledge of their strengths and limitations is key to avoiding misinterpretation and misuse.

Procalcitonin-guided therapeutic decisions have been linked to decrease in antimicrobial therapy duration; however, no clear mortality benefit has been found.

Procalcitonin study limitations such as low protocol adherence, high risk of bias, and longer antimicrobial therapy duration in non-procalcitonin groups call for future well-structured trials.

Modified pharmacokinetics in the critically ill, such as augmented renal clearance and increased volume of distribution, pose as risks for suboptimal antimicrobial therapy and therapeutic failure.

\section{DIGITAL FEATURES}

This article is published with digital features, including a summary slide, to facilitate understanding of the article. To view digital features for this article go to https://doi.org/10.6084/ m9.figshare.13169528.

\section{INTRODUCTION}

Infections are not only common causes of intensive care unit (ICU) admission but also frequent culprits of ICU-associated complications [1-3]. Although the beneficial aspects of antimicrobial therapy (AMT) are well acknowledged, the potentially associated harms are often underestimated [4]. Moreover, recent data indicate that about $70 \%$ of ICU patients are undergoing AMT [1] and up to $60 \%$ of these prescriptions are inadequate [5-8]. Naturally, antimicrobial stewardship programs (ASP) should constitute a pivotal part of ICU therapeutic decision-making.

The World Health Organization has declared antimicrobial resistance (AMR) a global health security threat, requiring immediate action [9]. In the face of rising AMR and elevated clinical and economic burdens of antimicrobial usage in the ICU, the regular implementation of ASP must be encouraged. Despite the elevated prevalence of infectious disease and clinicians' experience, diagnosis remains challenging as there is no gold standard diagnostic test. This has turned the spotlight onto biomarkers as promising adjunctive tools in ASP [10, 11]. Pharmacokinetic (PK) and pharmacodynamic (PD) issues concerning the critically ill and adequate therapeutic drug monitoring (TDM) should be taken into consideration when implementing ASP strategies [12].

This review provides an update on ASP, namely regarding its importance in the ICU and the roles biomarkers, PK, and PD play in ASP implementation.

\section{METHODS}

This article is based on previously conducted studies and contains previous studies performed by Pedro Póvoa and Luís Coelho. The authors conducted an updated review article focusing on the most recent evidence in the ICU setting. Preference was given to randomized controlled trials, although these are scarce amongst certain aspects of ASP, such as antimicrobial de-escalation. Relevant updated guidelines and respective quality of evidence were also discussed. The review of potential biomarkers was centered on the most widely studied-procalcitonin and C-reactive protein. 


\section{ANTIMICROBIAL STEWARDSHIP: WHAT IS THE PROBLEM?}

In the ICU setting, AMR is a widely acknowledged problem, justifying the crucial need for optimal AMT usage. Affected patients are at increased risk for worse clinical outcomes, mortality, and higher consumption of healthcare resources $[4,13]$.

In cases of severe infections, early administration of AMT is proven to lower mortality [14]. On the basis of this benefit, Surviving Sepsis Campaign (SSC) guidelines strongly recommend early initiation of AMT [15]. The risk of missing the potential window of opportunity frequently leads to a "safe path" of antimicrobials [16]. In addition to AMT timing [17], its adequacy is equally important. Patients with septic shock, for example, exhibit higher mortality rates with inadequate empirical regimens [18-22].

The well-acknowledged need for AMT and its impact on patient outcomes naturally hinder the implementation of ASP. The critically ill patient is the highest per-capita antimicrobial consumer [1, 4]. Moreover, critical illness itself, risks of organ support, and inherent immunosuppression of the majority of ICU patients all confer a predisposition for infectious complications [2] as well as unpredictable changes in $\mathrm{PK}$ and PD of AMT.

Current evidence advocates shorter AMT courses without negative impact on clinical outcomes in different infections and clinical settings [23-27]. However, limitations, such as frequently insufficient microbiological information, can make therapeutic accuracy and duration questionable aspects of care [28].

\section{Antibiotic Stewardship Programs in ICU}

The large burden of AMT consumption in the ICU setting should motivate intensivists to become active participants in developing, implementing, and promoting ASP. These should strive to reduce antibiotic consumption, resistance, and adverse side effects, whilst improving patient outcomes and economic burden [2, 29]. A team approach and use of clinical decision support systems are helpful adjuncts for ASP improvement.

\section{Antimicrobial De-escalation}

Antimicrobial de-escalation (ADE) has been encouraged as a strategy for ASP since 2001, with hopes of decreasing harm from broadspectrum AMT as well as pressure for AMR emergence [30]. SSC guidelines recognize the importance of $\mathrm{ADE}$, advocating its daily assessment, discontinuation of combination therapy, and narrowing AMT spectrum if clinical improvement or signs of infection resolution exist [15].

Unfortunately, ADE in the ICU remains suboptimal. The DIANA study, a prospective observational study, recently assessed ADE in adult ICUs of 28 countries. Only a minority of patients were subject to $\mathrm{ADE}$ on day 3 of empirical AMT even though combination therapy and carbapenems were frequently prescribed [31]. Severity of illness was higher than in previous studies that reported more frequent ADE [32], possibly justifying the lower ADE rate. Although clinical cure on day 7 (primary outcome) was higher in the ADE cohort, no impact on ICU and hospital length of stay (LOS) or 28-day mortality was found. The observational nature of the study cannot account for all confounding factors affecting therapeutic decisions and primary outcomes [31].

ADE remains a controversial topic, with available literature comprised essentially of observational studies and one small randomized controlled trial (RCT) [18, 33]. Although probably safe, selection bias prevails as clinicians apparently resort more to ADE in patients with favorable clinical progress [29]. Moreover, even the definition of ADE remains unclear and its impact on AMR poorly established $[18,33]$.

Leone et al. were the first to test ADE in an RCT. The ADE group showed longer durations of AMT, most likely related to the higher prevalence of superinfections in the group. Although the incidence of organ failure was not superior and mortality not affected, treatment was not blind and ADE definition arbitrary [34]. Posteriorly, De Bus et al. also associated ADE 
with fewer AMT-free days and longer ICU LOS [35].

In the setting of conflicting evidence, the European Society of Intensive Care Medicine and European Society of Clinical Microbiology and Infectious Diseases recently reviewed existing evidence on ADE and its effects on the critically ill. Thirteen recommendations were published, although many associated with low quality of evidence. ADE was deemed most likely safe regarding outcomes, but linked to a risk of increased total duration of AMT. Consequently, although both the duration of AMT and ADE should integrate ASP strategies, their assessment should be made separately. Ideally, ADE should occur within $24 \mathrm{~h}$ of culture and antibiogram results. No recommendation was made regarding the effect of ADE on AMR, use of biomarkers when considering ADE or TDM, and improved patient outcomes [33].

ADE can provide an unjustified sense of security with the assumption that short courses of empirical AMT do not impact AMR [18]. However, the ecological consequences of broadspectrum AMT start within days of first therapeutic courses [36]. Caution should be exercised to prevent indiscriminate choices of broadspectrum AMT since this is not innocuous [18].

\section{BIOMARKERS}

Diagnostic workup for infections can be challenging and delayed AMT initiation leads to increased mortality in severe infections [14]. In both community (CAP) and hospital-acquired pneumonia (HAP), for example, clinical and radiologic criteria have poor sensitivity and specificity. Moreover, microbiologic documentation is often delayed [21, 37, 38]. Diagnostic hardships have encouraged clinicians to seek objective solutions; and biomarkers, namely those of the inflammatory cascade, have been sought as surrogate markers of infection [11].

AMT guidance with biomarkers can promote treatment individualization, thereby preventing nonessential antimicrobial exposure and reducing treatment failure rates [23]. In the setting of infectious diseases, they should be easily available for routine clinical use and provide diagnostic, prognostic, and therapy follow-up characteristics [28].

Their potential roles in initiation and discontinuation of AMT have been sought to improve ASP. Some biomarkers are hard to implement in an acute emergency care setting. C-reactive protein (CRP) and procalcitonin (PCT) comprise the few biomarkers with adequate characteristics for clinical use in acute infections $[11,28]$, although diagnostic accuracy is not optimal and neither possesses specificity for source of infection [37, 39]. CRP can present a more attractive low-cost alternative to PCT, considering the high testing costs of the latter [23].

\section{Role in Predicting Infection, Diagnosis, and Initiation of Antimicrobial Therapy}

Matson et al. were the first to point out that serial measurements of CRP could alert clinicians to an elevated risk of infection. A 25\% increase in CRP concentration, in the absence of non-infectious causes, was highly suggestive of infection [40]. Few studies since then have focused on the role of biomarkers before the timing of diagnosis. Those that do exist are dedicated to respiratory infections, namely HAP [10]. One early observational study by Póvoa et al. identified CRP patterns of progression with different clinical courses and correlations with infections in the ICU (Table 1). A daily increase in CRP greater than $4.1 \mathrm{mg} / \mathrm{dL}$ was very suggestive of infection, with a greater predictive value for infection upon CRP concentrations of $8.7 \mathrm{mg} / \mathrm{dL}$. Such data further emphasizes that objective information obtained from longitudinal kinetics of biomarkers could greatly aid the clinician in decision-making, namely regarding AMT initiation and culture sampling [41]. Also in the recent BioVAP study, serial CRP values were found useful for predicting ventilator-associated pneumonia (VAP) during the first 6 days of mechanical ventilation while PCT kinetics were not [42].

Various studies have analyzed PCT's role in distinguishing bacterial pneumonia from other underlying etiologies at the time of diagnosis and consequently guiding AMT use. In fact, 


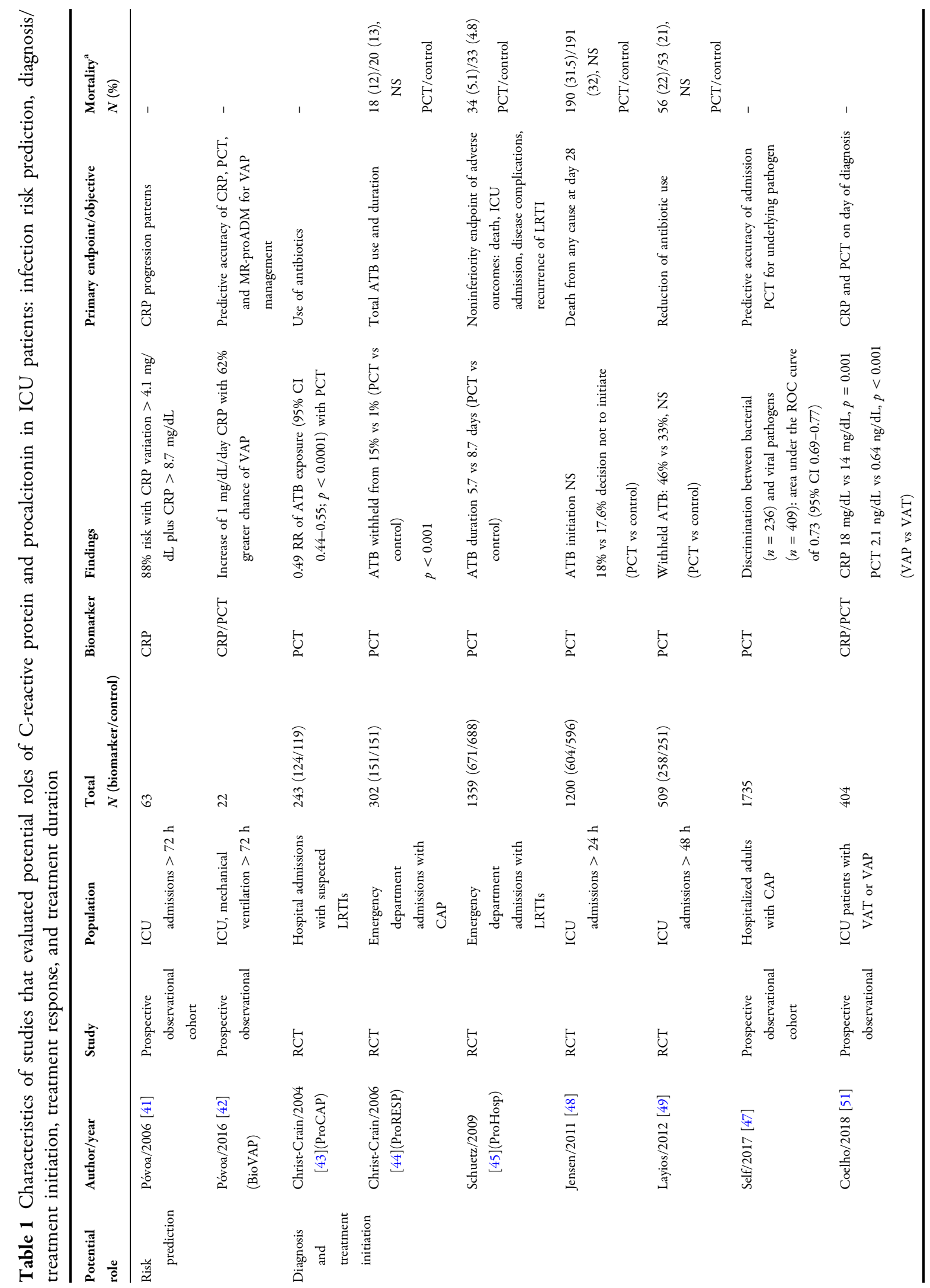




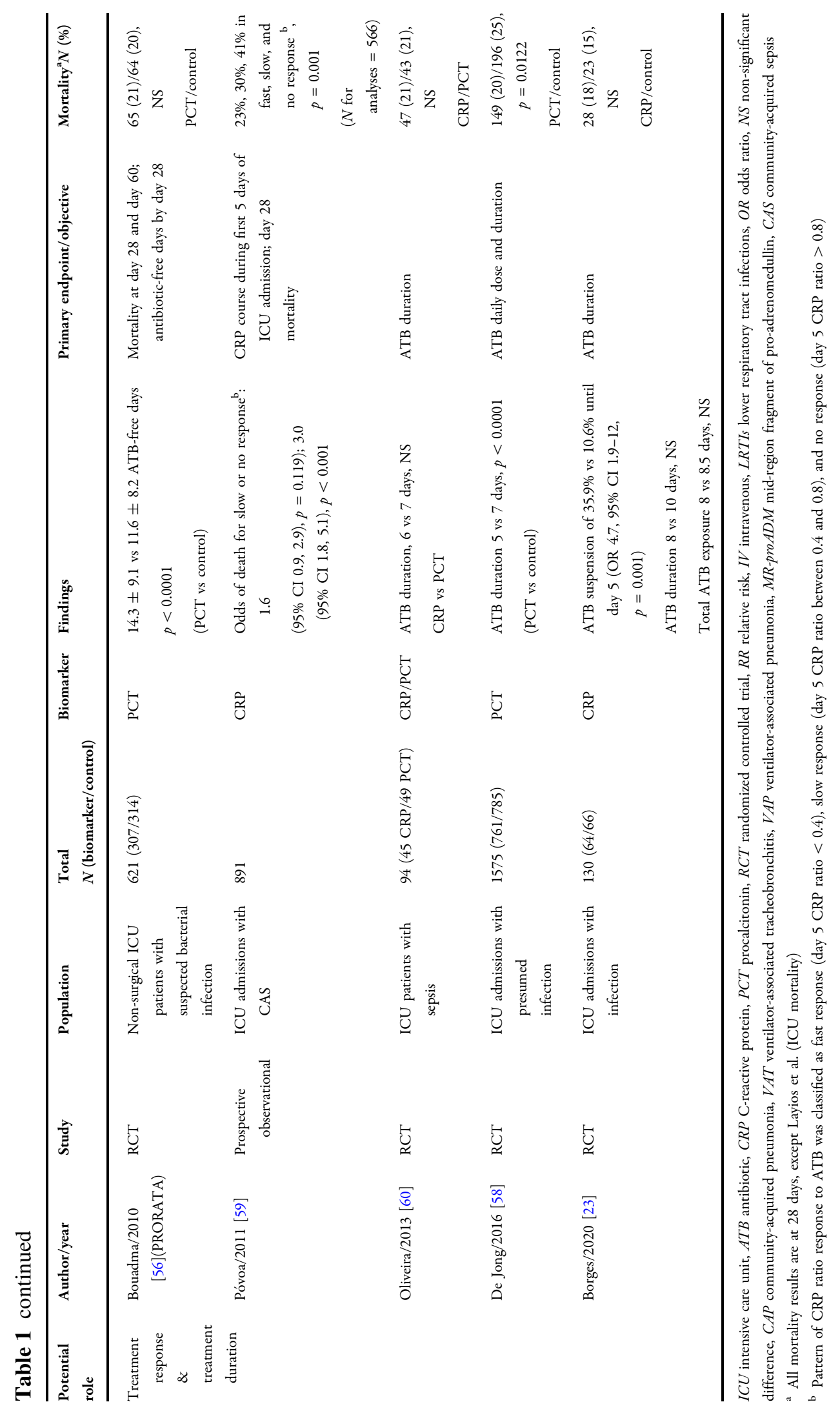


early research of the randomized studies ProCAP, ProRESP, and ProHosp, pointed to a reduction in AMT prescription with the use of PCT. However, an underlying risk of false negative results with low PCT levels was found as well [43-45]. The CAPNETZ study also found a high rate of overlap of PCT values between bacterial and non-bacterial etiologies [46]. Recently Self et al., in a diagnostic accuracy cohort study, evaluated PCT as a marker of CAP etiology in hospitalized adult patients. No reliable cutoff distinguished bacterial from viral causes, with the risk of not starting AMT in CAP. However, generalization can be hampered by some limitations, such as the impossibility of PCT availability in about one-fifth of patients, PCT measurements at admission instead of serial values, and detection of pathogens not necessarily responsible for infection [47].

Regarding PCT's impact on AMT initiation, Jensen et al. sought to evaluate whether a PCTcomplemented AMT escalation algorithm could reduce time to initiate appropriate AMT and improve survival in the critically ill, based on the previously developed concept of "alert PCT" $^{\prime}$. No mortality benefit was found and ICU LOS was increased [48]. Posteriorly, Layios et al. also evaluated a PCT-based algorithm for ASP in the ICU. No reduction in AMT exposure was attained and PCT did not distinguish probable, possible, or no infection upon AMT initiation with an area under the curve of 0.69 [49].

Kamat et al., in a recent meta-analysis, found that PCT level sensitivity and specificity were insufficient ( 0.55 and 0.76 , respectively) to distinguish viral from bacterial pneumonia, and consequently to either promote or discourage AMT initiation in cases of CAP [50].

A recent study by Coelho et al. provided analysis of the prospective multinational TAVeM database to differentiate VAP from ventilator-associated tracheobronchitis using CRP and PCT. Higher values of both biomarkers were observed in VAP. However, overlap of values in both infections refuted the biomarkers' discriminatory capacity between these two ventilator-associated lower respiratory tract infections with different severity [51].

Although these biomarkers can assist clinicians with the aforementioned advantages, clear knowledge of their limitations is essential to prevent misuse and misinterpretation. Méndez et al. recently related the inflammatory profile of patients with CAP at the time of diagnosis to the time of symptom onset. CRP levels were significantly lower in early presenters compared to PCT, interleukin-6 (IL-6), and IL-8 levels. On the other hand, these were significantly lower in non-early presenters while CRP levels remained increased [52].

\section{Assessment of Treatment Response and Impact on Duration of Antimicrobial Therapy}

PCT appears to have a favorable impact on AMT discontinuation and to be safe in patients with sepsis [53]. However, its impact on mortality is based on conflicting findings [54]. Various RCTs associate PCT with a decreased duration of AMT, albeit with some limitations such as low algorithm compliance, high rate of patient exclusion, possibly higher mortality and late organ failure in PCT arms, long and sometimes fixed duration of AMT in control groups, and disregard for renal failure effect on PCT levels $[11,55]$.

In the PRORATA trial, although mortality was similar in both PCT and control groups, antibiotic exposure was reduced in the PCT group. However, clinicians were free to initiate or end AMT irrespective of algorithm indications, reflecting low protocol compliance [56].

A recent meta-analysis conducted by Pepper et al. reviewed PCT-guided AMT discontinuation and mortality in the ICU adult patient [54]. Only one RCT designated mortality as a primary outcome and no significant decrease in mortality was found [57]. Improved survival and decreased duration of AMT were essentially observed in studies with low protocol adherence and algorithms incorporating CRP. De Jong et al. found a significant decrease in mortality associated with PCT-guided AMT discontinuation; however, survival benefit was not clearly linked to management choices influenced by PCT monitoring [58]. ASP implementation was not reported in any of the control groups [54]. The high risk of bias and low- 
certainty evidence calls into question the aforementioned benefits with PCT guidance. Consequently, guidelines do not presently recommend AMT decisions based solely on PCT values [38].

CRP can be a valuable marker of sepsis and AMT response. Póvoa et al. evaluated the patterns of serial CRP values in patients with sepsis over a 5-day period. The CRP ratio response to AMT was associated with clinical response and prognosis. Survivors showed an evident decrease in CRP values that was significantly lower than those of nonsurvivors. Furthermore, patients with no CRP ratio response had three times the odds of dying when compared to patients with a fast response [59].

Studies of CRP-guided algorithms are scarce, namely in the adult ICU population [23]. Oliveira et al. compared PCT and CRP guidance among ICU patients with sepsis in a singlecenter study. Both were considered equally useful in AMT reduction but no significant difference regarding AMT duration was found [60]. However, the maximum duration of AMT was stipulated as 7 days regardless of biomarker levels, reflecting a double trigger algorithm (biomarker kinetics or duration of AMT).

In a recent RCT, Borges et al. tested the impact of CRP-based protocols on AMT duration. The intervention group presented lower AMT exposure, with a higher percentage of AMT suspension up to the fifth day of followup. However, lower AMT exposure was only related to the index infection episode. The findings did not reveal a benefit in total AMT exposure or antibiotic-free days [23].

Von Dach et al., in an RCT, evaluated CRPguided AMT duration compared to 7-day and 14-day AMT regimens for Gram-negative bacteremia. The CRP-guided duration and fixed 7 -day group were noninferior to the 14-day group, with regards to 30-day clinical failure rate. However, limitations of result interpretation exist due to a large non-inferiority margin, low event rate, wide range of treatment duration in the CRP group (median 7 days), and low adherence rate. Although not centered on ICU admissions, findings reinforce that the patient's response, instead of predetermined fixed duration AMT regimens, should guide ASP decisions [61].

\section{Barriers to PCT Implementation}

Although results regarding PCT use have been promising, barriers to its regular implementation still exist. The costs associated with test realization, and consequently test availability, are one aspect to consider. De Jong et al. in the SAPS trial found a mean reduction of $€ 35$ per patient in AMT expenses. The AMT cost benefits could surpass PCT testing costs if these correspond to $€ 4$ or less, a price still far from current realities [58].

Effectively implemented ASP with clear protocols should facilitate PCT use in daily clinical practice. However, as the aforementioned studies have revealed, many PCT protocols leave the clinician at liberty to determine AMT duration, irrespective of protocol thresholds, reflecting low protocol compliance. Studies such as the PRORATA and SAPS trials witnessed protocol overruling in more than $50 \%[56,58]$, reflecting that clinical judgement frequently prevails.

\section{Biomarkers in Progress}

VAP is a common hospital-acquired infection associated with high mortality. Considering the hardships associated with clinical variables for diagnosis, bronchoalveolar lavage fluid (BALF) integrates the diagnostic workup for VAP. However, definite microbiologic results are often available only after AMT has been initiated. Conway Morris et al., in a prospective observational cohort study, evaluated the profile of serum and BALF inflammatory markers in patients with suspected VAP. The levels of IL-1 $\beta$ and IL-8 showed high sensitivity (almost 100\%) and consequently high negative predictive value [62].

Transcriptomics and metabolomics are currently under study as approaches for characterizing bacterial DNA and metabolites of the host or pathogen. Although various limitations exist and studies are scarce, volatile organic compounds (VOCs) obtained through non-invasive 
testing could provide promising tools for the confirmation or exclusion of infection. Various VOCs have been linked to the presence of specific bacterial strains [63, 64]. Through pathogen identification and discrimination of underlying activity, distinguishing between active infection and colonization could be possible [37]. Potential roles of various biomarkers in sepsis are currently under investigation, but substantial evidence is still lacking [11].

\section{PHARMACOKINETICS AND PHARMACODYNAMICS OF THE ICU PATIENT IN ANTIMICROBIAL STEWARDSHIP}

Critical illness accentuates physiological fluctuations, often due to organ dysfunction provoked by underlying acute illness, exacerbated chronic illness, and necessary interventions $[12,65]$. Efforts to achieve specific PK/PD targets may help decrease risk of therapeutic failure and AMR [65].

\section{Pharmacokinetics}

PK parameters $[65,66]$, together with dosage strategy, determine drug serum concentrations and consequently concentrations in tissues and body fluids [66]. The modified PK in the ICU patient, namely in terms of altered volume of distribution $\left(V_{\mathrm{d}}\right)$ and drug clearance $(\mathrm{Cl})$, challenge ASP optimization, encouraging AMT dosage individualization [7, 12]. Intravenous fluid loading, hypoalbuminemia, and endothelial dysfunction with consequent capillary leakage contribute to interstitial space expansion in the critically ill. Diseases with exuberant systemic inflammation accentuate this effect, such as burn victims and patients with sepsis. In the case of hydrophilic AMT (beta-lactams, aminoglycosides, daptomycin, glycopeptides), this can potentiate $V_{\mathrm{d}}$ expansion [67]. For protein-bound AMT (ceftriaxone, ertapenem, flucloxacillin, daptomycin), an increase in their unbound fraction, due to hypoalbuminemia, causes greater distribution and $\mathrm{Cl}$ [68].
Clinicians are vigilant regarding decreased $\mathrm{Cl}$ in renal impairment and necessary AMT adjustments. However, many critically ill patients demonstrate increased $\mathrm{Cl}$ despite normal serum creatinine values [69]. Udy et al. were the first to introduce the concept of augmented renal clearance (ARC) in the critically ill [70]. ARC is present in up to $80 \%$ of ICU patients, thought to occur after an acute insult. Those at risk are usually younger (less than 50 years old), of male gender, with lower severity scores and a history of trauma. A cutoff of creatinine clearance of greater than $130 \mathrm{~mL} / \mathrm{min} / 1.73 \mathrm{~m}^{2}$ is the most widely accepted, ideally obtained with a 8-24 h urine collection [69]. ARC poses the risk of suboptimal AMT concentrations [71] and subsequent treatment failure. Nevertheless, the true implications on patient outcomes are unclear [72].

Critically ill patients may require renal replacement therapy (RRT) for acute kidney injury. Extracorporeal drug handling may vary considerably depending on various factors (mode of RRT, filter porosity, protein binding, molecular weight, blood flow and effluent rates, etc.) [73]. $\mathrm{Cl}$ will depend essentially on the dialysis dose of RRT and eventual residual native renal function [74].

\section{Pharmacodynamics}

The PD of a drug reflects parameters of antimicrobial activity [75]. PD links PK exposure (serum concentration) to the pharmacological (capacity to kill or inhibit microbial growth) and toxicological effects of the drug [12, 76]. The pattern of antimicrobial activity over time is an important factor for effective dosage regimens [75].

Concentration-dependent patterns of bactericidal activity are observed with fluoroquinolones, aminoglycosides, and metronidazole's effect on anaerobic bacteria [77-79]. Time-dependent patterns rely on minimal concentration-dependent activity. Bactericidal saturation occurs at low multiples of minimal inhibitory concentrations (MIC). Therefore, concentration increases above these values (four to five times MIC) do not result in 
faster or more extensive bactericidal activity because of its dependence on the time of AMT exposure. This pattern is observed in beta-lactams, clindamycin, vancomycin, and macrolides. Consequently, dosage optimization implies increasing duration of time serum levels exceed the MIC, since regrowth of organisms begins soon after serum concentrations are below the MIC. On the contrary, for aminoglycosides and fluoroquinolones, the main pharmacokinetic/pharmacodynamic patterns related to AMT efficiency are peak/MIC and/or AUC/MIC ratios, reflecting concentration maximization [76].

\section{Antibiotic Dosing and Monitoring in ICU}

Increased knowledge of PK/PD, drug dosing, and AMR in the critically ill has shifted the rationale of TDM. Its importance goes beyond the intent to minimize toxicity in drugs with narrow therapeutic indices and complex PK [12]. TDM can be a reliable method for individualization and optimization of AMT dosing [65].

Adequate TDM may improve AMT exposure. However, suboptimal exposure may persist because of PK alterations, and delays in results can postpone dosage adjustments and clinical impact $[12,80,81]$. TDM is justified for antimicrobials with the following characteristics: significant intra- and/or inter-individual PK variability; defined exposure ranges that evoke pharmacological responses (clinical response and toxicity); defined sampling points; accurate and timely bioanalytical assay methods for drug dosing [12].

Mouton et al. pointed out that single MIC measurements can be unreliable because of variations in MIC. Inherent assay variations exist, compromising accuracy and reproducibility. Suboptimal dosing is a concern if the single MIC value corresponds to the low end of multiple MIC measurements [82]. Theoretically, when the MIC increases, PK exposure should also increase to guarantee an optimal PK/PD index. In the critically ill, this can be relevant since underlying pathogens can exhibit higher MICs [83]. Table 2 shows important PK and PD characteristics regarding the antibiotics for which TDM is currently recommended [12].

PK variability can challenge the accuracy of PK/PD indices. Beta-lactams, the most commonly prescribed AMT in ICUs, are hydrophilic drugs with consequent extracellular distribution, a small $V_{\mathrm{d}}$, and renal excretion. Risk of sub-therapeutic concentrations with increased $V_{\mathrm{d}}$ and ARC could be overcome with prolonged infusions of AMT. Regarding the main parameters of PK, absorption and metabolism are issues of little concern since in critically ill patients beta-lactam administration is IV and excretion is renal [65]. As previously mentioned, betalactams are time-dependent and so the ideal PK/ PD target should involve the time unbound drug concentration remains above the MIC (\%fT > MIC). Nevertheless, several studies discriminate different levels for the same beta-lactams to improve clinical outcomes $[7,65,84,85]$. Roberts et al. first demonstrated that prolonged infusions of beta-lactams led to higher drug concentrations $(100 \% \mathrm{fT}>\mathrm{MIC})$ and consequently higher likelihood of a positive clinical outcome [7]. However, only a minority of the patients with microbiologic results had MIC measurements and none of the $\mathrm{PK} / \mathrm{PD}$ of concomitant antimicrobials was assessed.

Unexplained PK variability, both inter- and intra-individual, is frequent and should be accounted for. TDM-based dosing adjustment is useful when inter-individual variability prevails since intra-individual variability can be overcome with covariate-based strategies [12]. Trough sampling constitutes the usual PK sampling for TDM, evaluating a single drug concentration against a therapeutic target. This is the easiest method but also the least accurate. Nomograms can be superior to conventional dosing, integrating PK/PD indices with parameters of organ function $[86,87]$. Nonetheless, to include additional covariates such as weight, single sampling is insufficient, and the timing of sampling must be predefined and strictly complied with.

Although for most antimicrobials TDM appears beneficial, namely with aminoglycosides, definitive data regarding the impact on clinical outcomes is scarce [12]. Abdulla et al. 
Table 2 Pharmacokinetic and pharmacodynamic characteristics regarding antibiotics for which therapeutic drug monitoring is currently recommended [12]

\begin{tabular}{|c|c|c|c|c|}
\hline Antimicrobial class & Monitoring & Sampling & Target & Toxicity threshold \\
\hline Aminoglycosides & $\begin{array}{l}\text { AUC-based } \\
C_{\max } / \mathrm{MIC} \\
C_{\min }\end{array}$ & $\begin{array}{l}\text { Two } \\
\text { samples }^{\mathrm{a}} \\
\text { One } \\
\text { sample }^{\mathrm{b}} \\
\text { One sample }^{\mathrm{c}}\end{array}$ & $\begin{array}{l}\text { AUC } 80-120 \mathrm{mg} \mathrm{h} / \mathrm{L} \\
C_{\max } / \mathrm{MIC} \geq 8-10\end{array}$ & Nephrotoxicity/ototoxicity \\
\hline Gentamicin/tobramicin & & & $C_{\min }<0.5 \mathrm{mg} / \mathrm{L}$ & $C_{\min }>1 \mathrm{mg} / \mathrm{L}$ \\
\hline Amikacin & & & $C_{\min }<2.5 \mathrm{mg} / \mathrm{L}$ & $C_{\min }>5 \mathrm{mg} / \mathrm{L}$ \\
\hline Beta-lactams & $\begin{array}{l}C_{\mathrm{min}} \\
C_{\mathrm{ss}}\end{array}$ & $\begin{array}{l}\text { One sample } \\
\text { One } \\
\text { sample }^{\mathrm{d}}\end{array}$ & $\begin{array}{l}100 \% \mathrm{fT}>\mathrm{MIC} \\
C_{\mathrm{ss}}>\mathrm{MIC}\end{array}$ & Nephrotoxicity/neurotoxicity \\
\hline Penicillins & & & $50-100 \%$ fT > MIC & $\begin{array}{l}C_{\min }>361 \mathrm{mg} / \mathrm{L} \text { (piperacillin nephro-/ } \\
\text { neurotoxicity) }\end{array}$ \\
\hline Cephalosporins & & & $45-100 \%$ fT $>$ MIC & $C_{\min }>20 \mathrm{mg} / \mathrm{L}$ (cefepime neurotoxicity) \\
\hline Carbapenems & & & $50-100 \%$ fT > MIC & $\begin{array}{l}C_{\min }>44.5 \mathrm{mg} / \mathrm{L} \text { (meropenem nephro-/ } \\
\text { neurotoxicity) }\end{array}$ \\
\hline \multicolumn{5}{|l|}{ Glycopeptides } \\
\hline Vancomycin & $\begin{array}{l}\mathrm{AUC} / \mathrm{MIC} \\
C_{\mathrm{min}} \\
C_{\mathrm{ss}}\end{array}$ & $\begin{array}{l}\text { Two } \\
\text { samples } \\
\text { One sample } \\
\text { One } \\
\text { sample }^{\mathrm{d}}\end{array}$ & $\begin{array}{l}\operatorname{AUC}(0-24) / \\
\quad \mathrm{MIC} \geq 400 \\
C_{\mathrm{min}} \geq 15-20 \mathrm{mg} / \mathrm{L}^{\mathrm{g}} \\
C_{\mathrm{ss}} 20-25 \mathrm{mg} / \mathrm{L}\end{array}$ & $\begin{array}{l}\text { Nephrotoxicity } \\
C_{\min }>20 \mathrm{mg} / \mathrm{L}\end{array}$ \\
\hline Teicoplanin & $C_{\min }$ & One sample & $C_{\min } \geq 15-30 \mathrm{mg} / \mathrm{L}$ & No definite data \\
\hline Others & & & & \\
\hline Linezolid $^{f}$ & $C_{\min }$ & One sample ${ }^{c}$ & $C_{\min } 2-7 \mathrm{mg} / \mathrm{L}$ & $C_{\min }>7$ (hematological) \\
\hline
\end{tabular}

$A U C$ area under the concentration-time curve, $C_{\max } / M I C$ ratio of maximum drug concentration to minimum inhibitory concentration, $M I C$ minimum inhibitory concentration, $C_{\text {min }}$ trough drug concentration, $C_{\text {ss }}$ average steady-state drug concentration, $A U C$ $(0-24) / M I C$ ratio of the area under the concentration-time curve during a 24-h period to minimum inhibitory concentration

a One sample $30 \mathrm{~min}$ after the end of infusion and another $6-22 \mathrm{~h}$ after infusion

b 30 min after the end of infusion

c 30 min or just before next dosing

d One sample at any time point during the infusion

e $1 \mathrm{~h}$ after the end of infusion and another within 1-2 $\mathrm{h}$ of next infusion

${ }^{f}$ Sampling should occur $48 \mathrm{~h}$ after initiation

$\mathrm{g}$ For severe infections 
are currently conducting the DOLPHIN trial, a multicenter RCT to determine the efficacy and cost-effectiveness of beta-lactam and fluoroquinolone TDM, with ICU LOS as a primary outcome [88].

\section{CONCLUSIONS}

Although the importance of ASP is unquestionable in the ICU, implementation is far from ideal. PCT and CRP have proven to be readily available biomarkers for more objective assessment in the setting of acute illness. Although recent RCT focusing on CRP-guided and multiple studies of PCT-guided strategies found positive results regarding the reduction of AMT duration, biomarkers should not be the only factors used in therapeutic decision-making. Various study limitations render questionable levels of evidence regarding total AMT exposure and survival benefits. Nevertheless, biomarkers can be considered promising adjuncts for ASP. Knowledge of their strengths and limitations should guide their clinical application, ideally alongside a global clinical evaluation. Future studies on biomarker-guided therapy should focus on populations similar to those found in the ICU, namely regarding clinical severity and prolonged AMT duration. PK and PD parameters can help monitor AMT; however, evidence is lacking regarding the impact of TDM on clinical outcomes.

\section{ACKNOWLEDGEMENTS}

Funding. No funding or sponsorship was received for this study or the publication of this article.

Authorship. All named authors meet the International Committee of Medical Journal Editors (ICMJE) criteria for authorship for this article, take responsibility for the integrity of the work as a whole, and have given their approval for this version to be published.
Disclosures. Patrícia Moniz, Luís Coelho, and Pedro Póvoa declare that they have no conflict of interest.

Compliance with Ethics Guidelines. This article is based on previously conducted studies and contains previous studies performed by Pedro Póvoa and Luís Coelho.

Open Access. This article is licensed under a Creative Commons Attribution-NonCommercial 4.0 International License, which permits any non-commercial use, sharing, adaptation, distribution and reproduction in any medium or format, as long as you give appropriate credit to the original author(s) and the source, provide a link to the Creative Commons licence, and indicate if changes were made. The images or other third party material in this article are included in the article's Creative Commons licence, unless indicated otherwise in a credit line to the material. If material is not included in the article's Creative Commons licence and your intended use is not permitted by statutory regulation or exceeds the permitted use, you will need to obtain permission directly from the copyright holder. To view a copy of this licence, visit http:// creativecommons.org/licenses/by-nc/4.0/.

\section{REFERENCES}

1. Vincent JL, Rello J, Marshall J, et al. International study of the prevalence and outcomes of infection in intensive care units. JAMA. 2009;302(21):2323.

2. Fierens J, Depuydt PO, De Waele JJ. A practical approach to clinical antibiotic stewardship in the ICU patient with severe infection. Semin Respir Crit Care Med. 2019;40(04):435-46.

3. van Vught LA, Klouwenberg PMCK, Spitoni C, et al. Incidence, risk factors, and attributable mortality of secondary infections in the intensive care unit after admission for sepsis. JAMA. 2016;315(14):1469.

4. Arulkumaran N, Routledge M, Schlebusch S, Lipman J, Morris AC. Antimicrobial-associated harm in critical care: a narrative review. Intensive Care Med. 2020;46(2):225-35. 
5. Luyt C-E, Bréchot N, Trouillet J-L, Chastre J. Antibiotic stewardship in the intensive care unit. Crit Care. 2014;18(5):480.

6. Bergmans D. Indications for antibiotic use in ICU patients: a one-year prospective surveillance. J Antimicrob Chemother. 1997;39(4):527-35.

7. Roberts JA, Paul SK, Akova M, et al. DALI: defining antibiotic levels in intensive care unit patients: are current -lactam antibiotic doses sufficient for critically ill patients? Clin Infect Dis. 2014;58(8): 1072-83.

8. Kollef $\mathrm{MH}$. Optimizing antibiotic therapy in the intensive care unit setting. Crit Care. 2001;5(4): 189-95.

9. World Health Organization. Antimicrobial resistance: global report on surveillance. 2014. Geneva: WHO.

10. Póvoa P, Coelho L, Bos LJ. Biomarkers in pulmonary infections. Clin Pulm Med. 2019;26(4): 118-25.

11. Nora D, Salluh J, Martin-Loeches I, Póvoa P. Biomarker-guided antibiotic therapy-strengths and limitations. Ann Transl Med. 2017;5(10):208.

12. Abdul-Aziz MH, Alffenaar JWC, Bassetti M, et al. Antimicrobial therapeutic drug monitoring in critically ill adult patients: a position paper. Intensive Care Med. 2020;46(6):1127-53.

13. Neidell MJ, Cohen B, Furuya Y, et al. Costs of healthcare- and community-associated infections with antimicrobial-resistant versus antimicrobialsusceptible organisms. Clin Infect Dis. 2012;55(6): 807-15.

14. Kumar A, Roberts D, Wood KE, et al. Duration of hypotension before initiation of effective antimicrobial therapy is the critical determinant of survival in human septic shock. Crit Care Med. 2006;34(6):1589-96.

15. Rhodes A, Evans LE, Alhazzani W, et al. Surviving Sepsis Campaign: international guidelines for management of sepsis and septic shock: 2016. Intensive Care Med. 2017;43(3):304-77.

16. Depuydt PO, De Bus L, De Waele JJ. Reducing antibiotic use in the ICU: a time-based approach to rational antimicrobial use. Annual update in intensive care and emergency medicine. Cham: Springer; 2016. p. 15-23.

17. Pruinelli L, Westra BL, Yadav P, et al. Delay within the 3-hour Surviving Sepsis Campaign guideline on mortality for patients with severe sepsis and septic shock. Crit Care Med. 2018;46(4):500-5.
18. De Waele JJ, Schouten J, Beovic B, Tabah A, Leone M. Antimicrobial de-escalation as part of antimicrobial stewardship in intensive care: no simple answers to simple questions-a viewpoint of experts. Intensive Care Med. 2020;46(2):236-44.

19. Montero JG, Pizarraya AG, Ortega AE, Delgado EF, Sánchez JML. Adequate antibiotic therapy prior to ICU admission in patients with severe sepsis and septic shock reduces hospital mortality. Crit Care. 2015;19(1):302.

20. Campion M, Scully G. Antibiotic use in the intensive care unit: optimization and de-escalation. J Intensive Care Med. 2018;33(12):647-55.

21. Ewig S, Bauer T, Torres A. The pulmonary physician in critical care * 4: nosocomial pneumonia. Thorax. 2002;57(4):366-71.

22. Póvoa P, Coelho L, Almeida E, et al. C-reactive protein as a marker of ventilator-associated pneumonia resolution: a pilot study. Eur Respir J. 2005;25(5):804-12.

23. Borges I, Carneiro R, Bergo R, et al. Duration of antibiotic therapy in critically ill patients: a randomized controlled trial of a clinical and C-reactive protein-based protocol versus an evidence-based best practice strategy without biomarkers. Crit Care. 2020;24(1):281.

24. Chastre J, Wolff M, Fagon JY, et al. Comparison of 8 vs 15 days of antibiotic therapy for ventilator-associated pneumonia in adults: a randomized trial. JAMA. 2003;290(19):2588-98.

25. Eliakim-Raz N, Yahav D, Paul M, Leibovici L. Duration of antibiotic treatment for acute pyelonephritis and septic urinary tract infection-7 days or less versus longer treatment: systematic review and meta-analysis of randomized controlled trials. J Antimicrob Chemother. 2013;68(10): 2183-91.

26. Onakpoya IJ, Walker AS, Tan PS, et al. Overview of systematic reviews assessing the evidence for shorter versus longer duration antibiotic treatment for bacterial infections in secondary care. PLoS One. 2018;13(3):e0194858.

27. Tansarli GS, Mylonakis E. Systematic review and meta-analysis of the efficacy of short-course antibiotic treatments for community-acquired pneumonia in adults. Antimicrob Agents Chemother. 2018;62(9):e00635-e718.

28. Dupuy A-M, Philippart F, Péan Y, et al. Role of biomarkers in the management of antibiotic therapy: an expert panel review: I-currently available biomarkers for clinical use in acute infections. Ann Intensive Care. 2013;3(1):22. 
29. Mathieu C, Pastene B, Cassir N, Martin-Loeches I, Leone M. Efficacy and safety of antimicrobial deescalation as a clinical strategy. Expert Rev Anti Infect Ther. 2019;17(2):79-88.

30. Rello J, Paiva JA, Baraibar J, et al. International conference for the development of consensus on the diagnosis and treatment of ventilator-associated pneumonia. Chest. 2001;120(3):955-70.

31. De Bus L, Depuydt P, Steen J, et al. Antimicrobial de-escalation in the critically ill patient and assessment of clinical cure: the DIANA study. Intensive Care Med. 2020;46(7):1404-17.

32. Tabah A, Cotta MO, Garnacho-Montero J, et al. A systematic review of the definitions, determinants, and clinical outcomes of antimicrobial de-escalation in the intensive care unit. Clin Infect Dis. 2016;62(8):1009-17.

33. Tabah A, Bassetti M, Kollef MH, et al. Antimicrobial de-escalation in critically ill patients: a position statement from a task force of the European Society of Intensive Care Medicine (ESICM) and European Society of Clinical Microbiology and Infectious Diseases (ESCMID) Critically Ill Patients Study Group (ESGCIP). Intensive Care Med. 2020;46(2): 245-65.

34. Leone M, Bechis C, Baumstarck K, et al. De-escalation versus continuation of empirical antimicrobial treatment in severe sepsis: a multicenter non-blinded randomized noninferiority trial. Intensive Care Med. 2014;40(10):1399-408.

35. De Bus L, Denys W, Catteeuw J, et al. Impact of deescalation of beta-lactam antibiotics on the emergence of antibiotic resistance in ICU patients: a retrospective observational study. Intensive Care Med. 2016;42(6):1029-39.

36. Armand-Lefèvre L, Angebault C, Barbier F, et al. Emergence of imipenem-resistant gram-negative bacilli in intestinal flora of intensive care patients. Antimicrob Agents Chemother. 2013;57(3): 1488-95.

37. Póvoa P, Coelho L, Bos LDJ. New biomarkers for respiratory infections. Curr Opin Pulm Med. 2020;26(3):232-40.

38. Torres A, Niederman MS, Chastre J, et al. International ERS/ESICM/ESCMID/ALAT guidelines for the management of hospital-acquired pneumonia and ventilator-associated pneumonia. Eur Respir J. 2017;50(3):1700582.

39. Rabello LSCF, Pitrowsky MT, Soares M, Póvoa P, Salluh JIF. Novel biomarkers in severe communityacquired pneumonia. Rev Bras Ter Intensiva. 2011;23(4):499-506.
40. Matson A, Soni N, Sheldon J. C-reactive protein as a diagnostic test of sepsis in the critically ill. Anaesth Intensive Care. 1991;19(2):182-6.

41. Póvoa P, Coelho L, Almeida E, et al. Early identification of intensive care unit-acquired infections with daily monitoring of C-reactive protein: a prospective observational study. Crit Care. 2006;10(2):R63.

42. Póvoa P, Martin-Loeches I, Ramirez P, et al. Biomarker kinetics in the prediction of VAP diagnosis: results from the BioVAP study. Ann Intensive Care. 2016;6(1):32.

43. Christ-Crain M, Jaccard-Stolz D, Bingisser R, et al. Effect of procalcitonin-guided treatment on antibiotic use and outcome in lower respiratory tract infections: cluster-randomised, single-blinded intervention trial. Lancet. 2004;363(9409):600-7.

44. Christ-Crain M, Stolz D, Bingisser R, et al. Procalcitonin guidance of antibiotic therapy in community-acquired pneumonia: a randomized trial. Am J Respir Crit Care Med. 2006;174(1):84-93.

45. Schuetz P, Christ-Crain M, Thomann R, et al. Effect of procalcitonin-based guidelines vs standard guidelines on antibiotic use in lower respiratory tract infections. JAMA. 2009;302(10):1059.

46. Krüger S, Ewig S, Papassotiriou J, et al. Inflammatory parameters predict etiologic patterns but do not allow for individual prediction of etiology in patients with CAP: results from the German competence network CAPNETZ. Respir Res. 2009;10(1): 65.

47. Self WH, Balk RA, Grijalva CG, et al. Procalcitonin as a marker of etiology in adults hospitalized with community-acquired pneumonia. Clin Infect Dis. 2017;65(2):183-90. https://doi.org/10.1093/cid/ cix317.

48. Jensen JU, Hein L, Lundgren B, et al. Procalcitoninguided interventions against infections to increase early appropriate antibiotics and improve survival in the intensive care unit: a randomized trial. Crit Care Med. 2011;39(9):2048-58.

49. Layios N, Lambermont B, Canivet JL, et al. Procalcitonin usefulness for the initiation of antibiotic treatment in intensive care unit patients. Crit Care Med. 2012;40(8):2304-9.

50. Kamat IS, Ramachandran V, Eswaran H, Guffey D, Musher DM. Procalcitonin to distinguish viral from bacterial pneumonia: a systematic review and metaanalysis. Clin Infect Dis. 2020;70(3):538-42.

51. Coelho L, Rabello L, Salluh J, et al. C-reactive protein and procalcitonin profile in ventilator- 
associated lower respiratory infections. J Crit Care. 2018;48:385-9.

52. Méndez R, Menéndez R, Cillóniz $\mathrm{C}$, et al. initial inflammatory profile in community-acquired pneumonia depends on time since onset of symptoms. Am J Respir Crit Care Med. 2018;198(3): 370-8.

53. Lam SW, Bauer SR, Fowler R, Duggal A. Systematic review and meta-analysis of procalcitonin-guidance versus usual care for antimicrobial management in critically ill patients. Crit Care Med. 2018;46(5): 684-90.

54. Pepper DJ, Sun J, Rhee C, et al. Procalcitonin-guided antibiotic discontinuation and mortality in critically ill adults. Chest. 2019;155(6):1109-18.

55. Póvoa P, Salluh JIF. Biomarker-guided antibiotic therapy in adult critically ill patients: a critical review. Ann Intensive Care. 2012;2(1):32.

56. Bouadma L, Luyt CE, Tubach F, et al. Use of procalcitonin to reduce patients' exposure to antibiotics in intensive care units (PRORATA trial): a multicentre randomised controlled trial. Lancet. 2010;375(9713):463-74.

57. Bloos F, Trips E, Nierhaus A, et al. Effect of sodium selenite administration and procalcitonin-guided therapy on mortality in patients with severe sepsis or septic shock. JAMA Intern Med. 2016;176(9): 1266.

58. de Jong E, van Oers JA, Beishuizen A, et al. Efficacy and safety of procalcitonin guidance in reducing the duration of antibiotic treatment in critically ill patients: a randomised, controlled, open-label trial. Lancet Infect Dis. 2016;16(7):819-27.

59. Póvoa P, Teixeira-Pinto AM, Carneiro AH, Portuguese Community-Acquired Sepsis Study Group SACiUCI. C-reactive protein, an early marker of community-acquired sepsis resolution: a multicenter prospective observational study. Crit Care. 2011;15(4):R169.

60. Oliveira CF, Botoni FA, Oliveira CR, et al. Procalcitonin versus $\mathrm{C}$-reactive protein for guiding antibiotic therapy in sepsis. Crit Care Med. 2013;41(10): 2336-43.

61. von Dach E, Albrich WC, Brunel A-S, et al. Effect of C-reactive protein-guided antibiotic treatment duration, 7-day treatment, or 14-day treatment on 30-day clinical failure rate in patients with uncomplicated gram-negative bacteremia. JAMA. 2020;323(21):2160.

62. Conway Morris A, Kefala K, Wilkinson TS, et al. Diagnostic importance of pulmonary interleukin-1 and interleukin-8 in ventilator-associated pneumonia. Thorax. 2010;65(3):201-7.

63. Bos LDJ, Sterk PJ, Schultz MJ. Volatile metabolites of pathogens: a systematic review. PLoS Pathog. 2013;9(5):e1003311.

64. van Oort $\mathrm{P}$, de Bruin $\mathrm{S}$, Weda $\mathrm{H}$, Knobel $\mathrm{H}$, Schultz $\mathrm{M}$, Bos L. Exhaled breath metabolomics for the diagnosis of pneumonia in intubated and mechanically-ventilated intensive care unit (ICU)patients. Int J Mol Sci. 2017;18(2):449.

65. Williams P, Cotta MO, Roberts JA. Pharmacokinetics/pharmacodynamics of $\beta$-lactams and therapeutic drug monitoring: from theory to practical issues in the intensive care unit. Semin Respir Crit Care Med. 2019;40(04):476-87.

66. Craig WA. State-of-the-art clinical article: pharmacokinetic/pharmacodynamic parameters: rationale for antibacterial dosing of mice and men. Clin Infect Dis. 1998;26(1):1-10.

67. Gonçalves-Pereira J, Póvoa P. Antibiotics in critically ill patients: a systematic review of the pharmacokinetics of $\beta$-lactams. Crit Care. 2011;15(5): R206.

68. Roberts JA, Pea F, Lipman J. The clinical relevance of plasma protein binding changes. Clin Pharmacokinet. 2013;52(1):1-8.

69. Mahmoud S, Shen C. Augmented renal clearance in critical illness: an important consideration in drug dosing. Pharmaceutics. 2017;9(4):36.

70. Udy AA, Putt MT, Shanmugathasan S, Roberts JA, Lipman J. Augmented renal clearance in the intensive care unit: an illustrative case series. Int J Antimicrob Agents. 2010;35(6):606-8.

71. Udy AA, Varghese JM, Altukroni M, et al. Subtherapeutic initial $\beta$-lactam concentrations in select critically ill patients. Chest. 2012;142(1):30-9.

72. Udy AA, Dulhunty JM, Roberts JA, et al. Association between augmented renal clearance and clinical outcomes in patients receiving $\beta$-lactam antibiotic therapy by continuous or intermittent infusion: a nested cohort study of the BLING-II randomised, placebo-controlled, clinical trial. Int J Antimicrob Agents. 2017;49(5):624-30.

73. Choi G, Gomersall CD, Tian Q, Joynt GM, Freebairn R, Lipman J. Principles of antibacterial dosing in continuous renal replacement therapy. Crit Care Med. 2009;37(7):2268-82.

74. Roberts JA, Joynt GM, Lee A, et al. The effect of renal replacement therapy and antibiotic dose on antibiotic concentrations in critically ill patients: 
data from the multinational sampling antibiotics in renal replacement therapy study. Clin Infect Dis. 2020. https://doi.org/10.1093/cid/ciaa224.

75. Ebert SC, Craig WA. Pharmacodynamic properties of antibiotics: application to drug monitoring and dosage regimen design. Infect Control Hosp Epidemiol. 1990;11(6):319-26.

76. Craig WA, Middleton WS. Pharmacokinetic/pharmacodynamic parameters: rationale for antibacterial dosing of mice and men. Clin Infect Dis. 1998;26(1):1-10.

77. Shah PM, Junghanns W, Stille W. Dosis-WirkungsBeziehung der Bakterizidie bei E. coli, K. pneumoniae und Staphylococcus aureus. Dtsch Med Wochenschr. 1976;101(9):325-8.

78. Vogelman B, Craig WA. Kinetics of antimicrobial activity. J Pediatr. 1986;108(5):835-40.

79. Craig WA, Ebert SC. Killing and regrowth of bacteria in vitro: a review. Scand J Infect Dis Suppl. 1990;74:63-70.

80. Tabah A, De Waele J, Lipman J, et al. The ADMINICU survey: a survey on antimicrobial dosing and monitoring in ICUs. J Antimicrob Chemother. 2015;70(9):2671-7.

81. Wong G, Brinkman A, Benefield RJ, et al. An international, multicentre survey of $\beta$-lactam antibiotic therapeutic drug monitoring practice in intensive care units. J Antimicrob Chemother. 2014;69(5):1416-23.

82. Mouton JW, Muller AE, Canton R, Giske CG, Kahlmeter G, Turnidge J. MIC-based dose adjustment: facts and fables. J Antimicrob Chemother. 2018;73(3):564-8.
83. Sievert DM, Ricks P, Edwards JR, et al. Antimicrobial-resistant pathogens associated with healthcareassociated infections summary of data reported to the National Healthcare Safety Network at the Centers for Disease Control and Prevention, 2009-2010. Infect Control Hosp Epidemiol. 2013;34(1):1-14.

84. Jager NGL, van Hest RM, Lipman J, Taccone FS, Roberts JA. Therapeutic drug monitoring of antiinfective agents in critically ill patients. Expert Rev Clin Pharmacol. 2016;9(7):961-79.

85. McKinnon PS, Paladino JA, Schentag JJ. Evaluation of area under the inhibitory curve (AUIC) and time above the minimum inhibitory concentration ( $>$ MIC) as predictors of outcome for cefepime and ceftazidime in serious bacterial infections. Int J Antimicrob Agents. 2008;31(4):345-51.

86. Cristallini S, Hites M, Kabtouri H, et al. New regimen for continuous infusion of vancomycin in critically ill patients. Antimicrob Agents Chemother. 2016;60(8):4750-6.

87. Pea F, Viale P, Cojutti P, Furlanut M. Dosing nomograms for attaining optimum concentrations of meropenem by continuous infusion in critically ill patients with severe gram-negative infections: a pharmacokinetics/pharmacodynamics-based approach. Antimicrob Agents Chemother. 2012;56(12):6343-8.

88. Abdulla A, Ewoldt TMJ, Hunfeld NGM, et al. The effect of therapeutic drug monitoring of beta-lactam and fluoroquinolones on clinical outcome in critically ill patients: the DOLPHIN trial protocol of a multi-centre randomised controlled trial. BMC Infect Dis. 2020;20(1):57. 